Journal of OBjeCt TECHNOLOGY

Published by AITO - Association Internationale pour les Technologies Objets

http://www.jot.fm/

\title{
The 15th European Conference on Modelling Foundations and Applications (ECMFA)
}

\author{
Benoit Combemale $^{\mathrm{a}} \quad$ Shaukat $\mathrm{Ali}^{\mathrm{b}}$
}

a. University of Toulouse \& Inria, France

b. Department of Engineering Complex Software Systems, Simula Research Laboratory, Norway

We welcome all the participants of the $15^{\text {th }}$ European Conference on Modelling Foundations and Applications (ECMFA 2019), in Eindhoven, The Netherlands.

The European Conference on Modelling Foundations and Applications is a premium venue dedicated for achieving the following two key objectives: 1) advancing state of the art in the area of Model-Based Engineering (MBE); 2) promoting the practical applications of MBE. It is also aimed at engaging exciting and fruitful discussions among researchers and practitioners with the ultimate aim of facilitating the transfer of research results into practice for improving the quality of software. Last but the not the least, ECMFA also provide a platform for young researchers and students to receive feedback from the leading researchers and practitioners on their research.

Same as the previous editions, ECMFA 2019 featured two tracks: 1) Foundation Track: This track solicits papers reporting a novel and foundational research in MBE; 2) Applications Track: This track seeks papers reporting the practical applications of MBE including experience reports and industrial applications.

We received 22 submissions for the Foundation track, and 11 papers were accepted, i.e., an acceptance rate of $50 \%$. For the Application track, we received ten submissions for the main track, and four papers were accepted, i.e., an acceptance rate of $40 \%$. This lead to an overall acceptance rate for both tracks of $47 \%$.

In 2019, we decided to brings some novelties, and in particular all the accepted papers are published in platinum open-access as journal articles in the JOT journal ${ }^{1}$. This is an important change that turns the accepted papers in journal article, with great visibility thanks to open-access.

We also decided to create a common program with the International Conference on Model Transformation (ICMT), such as all the accepted papers of both conferences have been organized topic-wise within the sessions. The program of ECMFA 2019 also includes two outstanding keynotes given by Prof. Friedrich Steimann and Dr. Ivan Kurtev. Prof. Steimann from the University of Hagen, Germany gives a keynote about Fatal Abstraction. Dr. Kurtev from Altran, Eindhoven, The Netherlands gives

\footnotetext{
${ }^{1}$ cf. http://www.jot.fm
} 
a keynote on Engineering of Software Factories for High-tech Industry - Experience and Challenges.

Last but not least, to strengthen STAF 2019 as an essential modeling meet-up for the community, the program also includes a Research Project Showcase and a newly created Junior Researcher Community Event.

We would like to thank our Communication Chair, Erwan Bousse, for an excellent job in advertising the conference through many channels and keeping the website up to date. We would also like to thank our Program Committee members for their dedication in providing timely reviews of high scientific quality and taking active participation in the discussion. Without their significant efforts, it wasn't possible to produce an exciting proceeding.

We look forward to seeing you in Eindhoven, The Netherlands!

Benoit Combemale Shaukat Ali

June 14, 2019

\section{Organization}

\section{Program co-Chairs:}

Benoit Combemale, University of Toulouse, France (Foundation track)

Shaukat Ali, Simula, Norway (Application track)

\section{Communication Chair:}

Erwan Bousse, University of Nantes, France

\section{Program Committee:}

Anthony Anjorin, Paderborn University

Alessandra Bagnato, Softeam

Francis Bordeleau, Cmind Inc

Erwan Bousse, Université de Nantes / LS2N

Marco Brambilla, Politecnico di Milano

Jean-Michel Bruel, IRIT

Eric Cariou, LIUPPA / Université de Pau

Antonio Cicchetti, Mälardalen University

Federico Ciccozzi, Mälardalen University

Zinovy Diskin, McMaster University / University of Waterloo

Romina Eramo, University of L'Aquila

Michalis Famelis, University of Montreal

Sebastien Gerard, CEA, LIST

Sudipto Ghosh, Colorado State University

Martin Gogolla, Database Systems Group, University of Bremen

Jeff Gray, University of Alabama

Joel Greenyer, Leibniz Universität Hannover

Esther Guerra, Universidad Autónoma of Madrid

Øystein Haugen, Østfold University College and SINTEF

Regina Hebig, Chalmers / Gothenburg University 
Jennifer Horkoff, Chalmers and the University of Gothenburg

Jörg Kienzle, McGill University

Dimitris Kolovos, University of York

Thomas Kuehne, Victoria University of Wellington

Ralf Lämmel, Universität Koblenz-Landau

Timothy Lethbridge, University of Ottawa

Brice Morin, SINTEF ICT

Richard Paige, University of York

Bernhard Rumpe, RWTH Aachen University

Davide Di Ruscio, Università degli Studi dell'Aquila

Houari Sahraoui, University of Montreal

Markus Schacher, KnowGravity Inc.

Bran Selic, Malina Software Corp.

Massimo Tisi, IMT Atlantique / LS2N

Juha-Pekka Tolvanen, MetaCase

Javier Troya, University of Seville

Daniel Varro, McGill University / Budapest University of Technology and Economics

Andrzej Wąsowski, IT University Manuel Wimmer, TU Wien

Steffen Zschaler, King's College London

\section{Sub-reviewers:}

Edouard Batot, University of Montreal, Canada

Phillipa Bennett, The University of the West Indies, Jamaica

Alessio Bucaioni, Alessio Bucaioni, Sweden

Márton Búr, Budapest University of Technology and Economics, Hungary

Johannes Härtel, University of Koblenz, Landau, Germany

Marcel Heinz, University of Koblenz, Landau, Germany

Carlos Hernández Corbato, TU Delft, The Netherlands

Nico Jansen, RWTH Aachen University, Germany

Rodi Jolak, Chalmers University, Sweden

Andreas Kästner, University of Bremen, Germany

Jörg Christian Kirchhof, RWTH Aachen University, Germany

Dorian Leroy, Johannes Kepler University Linz, Austria Sina Madani, The University

of York, UK

Philipp Seifer, University of Koblenz, Landau, Germany

Mahsa Varshosaz, Högskolan i Halmstad, Sweden

Alfa Yohannis, The University of York, UK 